\title{
Sensitivity improved Cerenkov luminescence endoscopy using optimal system parameters
}

\author{
Xueli Chen ${ }^{1 \#}$, Xinyu Wang ${ }^{1 \#}$, Tianyu Yan ${ }^{1}$, Yun Zheng ${ }^{1}$, Honghao Cao ${ }^{1}$, Feng Ren ${ }^{1}$, Xu Cao ${ }^{1}$, \\ Xiangfeng Meng ${ }^{2 \#}$, Xiaojian $\mathrm{Lu}^{3}$, Shuhui Liang ${ }^{4}$, Kaichun $\mathrm{Wu}^{4}$ \\ ${ }^{1}$ Engineering Research Center of Molecular and Neuro Imaging of Ministry of China, School of Life Science and Technology, Xidian University, \\ Xi'an, China; ${ }^{2}$ Institute of Medical Device Control, National Institutes for Food and Drug Control, Beijing, China; ${ }^{3}$ Nanjing Chunhui Science and \\ Technology Industrial Co. Ltd., Nanjing, China; ${ }^{4}$ Fourth Military Medical University, State Key Laboratory of Cancer Biology and Xijing Hospital \\ of Digestive Diseases, Xi'an, China
}

Contributions: (I) Conception and design: X Chen, X Wang, X Lu, K Wu; (II) Administrative support: X Chen, X Lu, K Wu; (III) Provision of study materials or patients: X Cao, S Liang, K Wu; (IV) Collection and assembly of data: X Wang, T Yan, Y Zheng, H Cao; (V) Data analysis and interpretation: X Chen, X Wang, F Ren, X Meng; (VI) Manuscript writing: All authors; (VII) Final approval of manuscript: All authors.

\#These authors contributed equally to this work.

Correspondence to: Xueli Chen. Engineering Research Center of Molecular and Neuro Imaging of Ministry of Education \& School of Life Science and Technology, Xidian University, Xi'an 710071, China. Email: xlchen@xidian.edu.cn; Xiaojian Lu. Nanjing Chunhui Science and Technology Industrial Co. Ltd., Nanjing 210012, China. Email: 76159477@qq.com; Kaichu Wu. Fourth Military Medical University, State Key Laboratory of Cancer Biology and Xijing Hospital of Digestive Diseases, Xi’an 710032, China. Email: kaicwu@fmmu.edu.cn.

Background: The challenges of clinical translation of optical imaging, including the limited availability of clinically used imaging probes and the restricted penetration depth of light propagation in tissues can be avoided using Cerenkov luminescence endoscopy (CLE). However, the clinical applications of CLE are limited due to the low signal level of Cerenkov luminescence and the large transmission loss caused by the endoscope, which results in a relatively low detection sensitivity of current CLE. The aim of this study was to enhance the detection sensitivity of the CLE system and thus improve the system for clinical application in the detection of gastrointestinal diseases.

Methods: Four optical fiber endoscopes were customized with different system parameters, including monofilament (MF) diameter of imaging fiber bundles, fiber material, probe coating, etc. The endoscopes were connected to the detector via a specifically designed straight connection device to form the CLE system. The $\beta-2-\left[{ }^{18} \mathrm{~F}\right]$-Fluoro-2-deoxy-D-glucose $\left({ }^{18} \mathrm{~F}-\mathrm{FDG}\right)$ solution and the radionuclide of Gallium-68 $\left({ }^{68} \mathrm{Ga}\right)$ were used to evaluate the performance of the CLE system. The images of the ${ }^{18} \mathrm{~F}-\mathrm{FDG}$ solution acquired by the CLE were used to optimize imaging parameters of the system. By using the endoscope with optimized parameters, including the MF diameter of imaging fiber bundles, fiber materials, etc., the resolution and sensitivity of the assembled CLE system were measured by imaging the radionuclide of ${ }^{68} \mathrm{Ga}$.

Results: The results of ${ }^{18} \mathrm{~F}-\mathrm{FDG}$ experiments showed that larger MF diameter led to higher collection efficiency. The fiber material and probe coating with high transmission ratios in the range of 400-900 nm also increased signal collection and transmission efficiency. The results of ${ }^{68} \mathrm{Ga}$ evaluations showed that a minimum radioactive activity of radionuclides as low as $0.03 \mu \mathrm{Ci}$ was detected in vitro within 5 minutes, while that of $0.68 \mu \mathrm{Ci}$ can be detected within 1 minute. In vivo experiments also demonstrated that the developed CLE system achieved a high sensitivity at a submicrocurie level; that is, $0.44 \mu \mathrm{Ci}$ within 5 minutes, and 0.83 $\mu \mathrm{Ci}$ within 1 minute. The weaker in vivo sensitivity was due to the attenuation of the signal by the mouse tissue skin and the autofluorescence interference produced by biological tissues.

Conclusions: By optimizing the structural parameters of fiber endoscope and imaging parameters for data 
acquisition, we developed a CLE system with a sensitivity at submicrocurie level. These results support the possibility that this technology can clinically detect early tumors within 1 minute.

Keywords: Cerenkov luminescence imaging; endoscopy; sensitivity enhancement; monofilament fiber diameter; fiber material and probe coating

Submitted Apr 04, 2021. Accepted for publication Jul 06, 2021. doi: $10.21037 /$ qims-21-373

View this article at: https://dx.doi.org/10.21037/qims-21-373

\section{Introduction}

Gastric cancer is a highly prevalent disease in China and has the highest mortality rate in the world (1-3). Currently, the conventional diagnostic method for gastric cancer involves the use of white light endoscopy to observe tumor morphology change. However, the change of tumor morphology cannot be identified in the early stages of tumor development.

The molecular function of tumor cells changes earlier than that of tumor morphology. Thus, optical imaging, an important branch of molecular imaging technology, has become one of the most widely used techniques in the field, as it can quantify the living biological processes from the cellular and molecular levels (4-10) and may provide a solution to early diagnosis of gastric cancer. In particular, Cerenkov luminescence imaging, which is based on the Cerenkov radiation effect (11-13), can solve the problem of limited available probes for clinical applications of optical imaging techniques (14-20). The emergent Cerenkov luminescence endoscopy (CLE), the product of further combination with endoscopic detection techniques, can overcome the issue of limited penetration depth encountered in the optical imaging of gastric cancer. CLE therefore opens up new potential for the early diagnosis of gastric cancer.

The concept and prototype system of CLE, first developed by Cheng et al. $(21,22)$. involved a chargecoupled device (CCD) camera that could image the ocular head of a commercial endoscope. There was no physical link between the CCD camera and the endoscope, and the light transfer process between the 2 instruments occurred in free space. This strategy demonstrated the feasibility of the use of CLE in tumor recognition and surgical resection (22). Subsequently, Cao et al. developed a CLE system that seamlessly integrated an electron multiplying charge-coupled device (EMCCD) camera with a clinical gastrofiberscope (23). The group used an optical adapter to complete a seamless link between the EMCCD and the endoscope. The minimum theoretical activity of Gallium-68 $\left({ }^{68} \mathrm{Ga}\right)$ that could be detected was $1.5 \mu \mathrm{Ci}$ for in vitro and $3.2 \mu \mathrm{Ci}$ for in vivo measurements within 5 minutes. The same system was also applied to the preliminary diagnosis of clinical colorectal cancer patients, yielding consistent diagnostic results with those obtained by positron emission tomography (PET) (24). Using similar techniques, Song et al. subsequently constructed a CLE system by combining the electron multiplying CCD with a rigid laparoscope and validated its effectiveness by performing imaging on a mouse abdominal cavity (25). Despite the effectiveness of the current CLE systems, their broader use has been limited, particularly within clinical settings. This is because it currently takes 5 minutes to capture a single high-quality image, and this long data acquisition time severely restricts the clinical usability and value of the system.

The long data acquisition time is a result of the poor sensitivity of the current CLE system. The two contributing factors include the weakness of the Cerenkov luminescent (CL) light emission and the large light loss of the endoscope. One method to overcome these limitations is to enhance the emission of luminescence light. Researchers have previously aimed to find more suitable radionuclides with a higher yield of CL light (26), while other studies have tried to enhance the CL light emission by using scintillators or combining the radionuclides with nanoparticles (27-35). An example of this was demonstrated in the study by Cao et al., where the detection sensitivity of the CLE system was improved by a factor of 50 (30). Although these are effective solutions, new nanoparticle probes and radionuclides with a high yield of CL light are generally not clinically available.

A second method for improving the system collection efficiency involves optimizing the detection performance of the CLE system. This can be achieved by designing the objective probe, fiber parameters, and materials of the optical fiber endoscope to be more suited to CL collection 


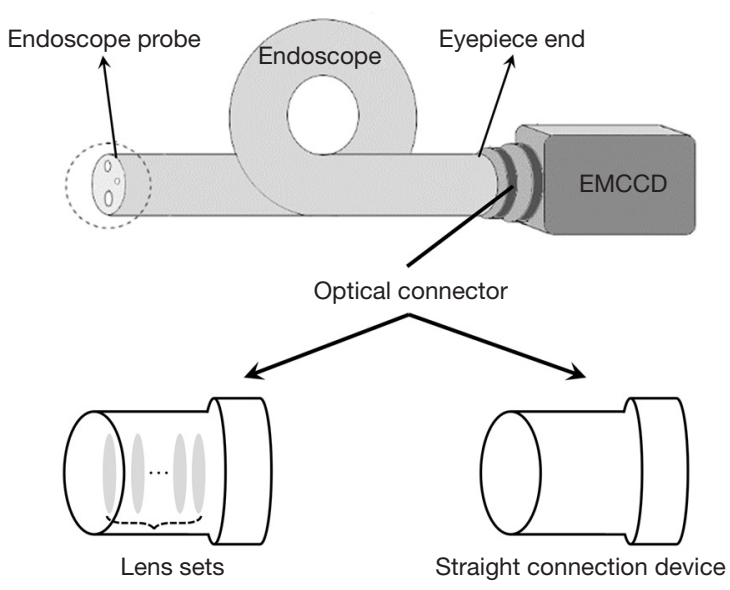

Figure 1 Schematic of the CLE system. (Lower left) The CLE system connected via the straight connection device. (Lower right) The CLE system connected via the converter lens. CLE, Cerenkov luminescence endoscope.

and transmission; designing an optical adapter for large light transmission efficiency; and selecting a detector suitable for CL light sensing.

This paper focuses on the second method of system-level optimization to enhance the detection sensitivity of the CLE system. We first investigated the influence of imaging parameters on detection performance and then developed a CLE system with high detection sensitivity. Through a series of experiments, we systematically evaluated the performance of the developed CLE system by measuring the in vitro and in vivo detection sensitivity, indicating the minimum radionuclide activity. The feasibility of clinical use of the system was determined based on these measurements of minimum activity.

\section{Methods}

\section{Design of the CLE system}

The CLE system presented in this study has different structures from those used in our previous studies $(24,25)$. An optical fiber endoscope was connected to the EMCCD (Andor iXon Ultra 888/897) camera via a straight connection device (SCD; Figure 1, lower left). The SCD was first used to adjust the distance between the eyepiece end of the endoscope and the EMCCD chip. The SCD was then used to avoid leakage of ambient light into the system. One end of the SCD was plugged directly into the EMCCD camera via the standard C-port, and the other end was connected to the eyepiece port of the endoscope via the standard interface. Both the standard C-port and the standard interface were tightly connected to the connected object and were not adjustable, which avoided the emergence of possible gaps that could cause ambient light leakage problems. In contrast to the converter lens (Figure 1, lower right), the SCD lacked a lens set, which acted to reduce the light loss attenuated by the lens set to a certain extent. In the developed CLE system, the optical fiber endoscope and the SCD were made in-house by Nanjing Chunhui Science and Technology Industrial Co. Ltd and the EMCCD was purchased from the Titan Electro-Optics Co. Ltd.

\section{Optical fiber endoscopes with different structural parameters}

In the developed CLE system, the optical fiber endoscope is made up of a fiber bundle and an endoscopic probe. The fiber bundle contains tens of thousands of monofilament (MF) fibers, while the endoscope probe is made up of an objective lens set, located at the front of the optical fiber endoscope (as labeled in Figure 1). The structural parameters mentioned above determine the performance of the optical fiber endoscope, which further affect the detection sensitivity of the CLE system. We therefore first optimized the structural parameters of the optical fiber endoscope. In theory, a larger range of pixel size in an EMCCD camera means a larger MF diameter and a better light-collecting ability of the fiber bundle. In the CLE experiment, the EMCCD camera usually operates in a binning mode, for example $4 \times 4$ binning. It was hypothesized that if set at $4 \times 4$ binning, the pixel size of the detector used in our system would be larger than $50 \mu \mathrm{m}$. To validate this hypothesis, we customized 3 endoscopes with different MF diameters, labeled A, B, and C in Table 1 . Using these endoscopes, we observed the influence of different MF diameters on the imaging results of CLE. The detailed structural parameters are shown in Table 1 .

The CL emission has a broad spectrum (13), ranging from ultraviolet to the near-infrared region. Such a broad spectral range has high requirements for the fiber materials of image bundles and probe coating. To investigate the influence of the fiber materials and probe coating, we customized another endoscope that had the same field of view (FOV) angle and $\mathrm{MF}$ diameter as endoscope $\mathrm{C}$ had; however, the custom endoscope was made from different fiber materials and probe coating (labeled D in Table 1). The detailed parameters are listed in Table 1. 
Table 1 Parameters of 4 endoscopes that were customized by the Nanjing Chunhui Science and Technology Industrial Co. Ltd.

\begin{tabular}{|c|c|c|c|c|}
\hline Parameters & $A$ & $\mathrm{~B}$ & $\mathrm{C}$ & $\mathrm{D}$ \\
\hline MF diameter $(\mu \mathrm{m})$ & 20 & 25 & 30 & 30 \\
\hline Material & F2 glass frit & F2 glass frit & F2 glass frit & Silica glass \\
\hline Fiber bundle diameter (mm) & 1.8 & 1.8 & 1.8 & 1.8 \\
\hline
\end{tabular}

FOV, field of view; MF, monofilament.

\section{Comparative evaluation of different optical fiber endoscopes}

In this experiment, $300 \mu \mathrm{Ci}$ of ${ }^{18} \mathrm{~F}$-FDG mixed with saline constitutes $300 \mu \mathrm{L}$ of mixed liquid filled a single well of a black 96-well plate which was placed directly below the endoscope probe to act as the emission source of CL light. Due to the fact that Cerenkov luminescence is susceptible to interference from ambient light, the well plate was placed together with the endoscope probe in a home-made lighttight box. The endoscope eyepiece end was connected to the EMCCD camera by the SCD. The EMCCD was left outside of the light-tight box, and the joint of the EMCCD and endoscope eyepiece end was wrapped by shading cloth. The room light in the data acquisition room was left off during the experimental data acquisition. The experimental configuration was set up to mimic the reality of clinical applications. The relevant results of the noise analysis (Figure S1) showed that the effect of ambient noise in such an experimental setup is negligible. In order to balance the efficiency of signal collection and clinical operability, the distance between the endoscope probe and ${ }^{18} \mathrm{~F}$-FDG source was fixed at $1 \mathrm{~cm}$. For each endoscope, 5 images were acquired in succession for further analysis. During data acquisition, the parameters of EMCCD were set as binning $=4 \times 4, \mathrm{EM}=1000$, and exposure time $=300 \mathrm{~s}$.

Because the MF diameter of the fiber bundle may affect the spatial resolution of the CLE system, we tested the white-light spatial resolution of the CLE system equipped with endoscopes of different MF diameters using a resolution test board (1951 USAF) as a sample. During data acquisition, the distance between the endoscope probe and the resolution test board was $1 \mathrm{~cm}$, and the EMCCD parameters were set as $\mathrm{EM}=1$, binning $=1 \times 1$, and exposure time $=0.1 \mathrm{~s}$.

\section{Influence of source-probe distance on signal detection}

The distance from radionuclides to endoscope probe [source-probe (SP) distance] greatly influences signal detection. We investigated the dependency of CLE signal intensity on SP distance. In the experiment, $300 \mu \mathrm{L}$ of the ${ }^{18} \mathrm{~F}$-FDG solution containing $150 \mu \mathrm{Ci}$ radionuclides was injected into a single well of a black 96-well plate. The initial SP distance was set at $0 \mathrm{~cm}$; i.e., the endoscope probe was immediately adjacent to the CL emission source. When the SP distance was within $1 \mathrm{~cm}$, the SP distance was increased every $0.2 \mathrm{~cm}$. Starting from $1 \mathrm{~cm}$, the endoscope probe was then moved upward, increasing the SP distance at intervals of $0.5 \mathrm{~cm}$ until the SP distance reached $5 \mathrm{~cm}$. At each distance, 3 CLE images were acquired in succession and were then averaged for further analysis. The EMCCD parameters were set as $\mathrm{EM}=1,000$, binning $=1 \times 1$, and exposure time $=120 \mathrm{~s}$. This SP distance affected the FOV detected by the optical fiber endoscope. When the FOV angle of the endoscope probe was $45^{\circ}$, the FOV detected by the endoscope was five-sixths of the SP distance. For example, when the $\mathrm{SP}$ distance was $1 \mathrm{~cm}$, the FOV size was $0.83 \mathrm{~cm}$.

\section{Performance evaluation of the proposed CLE system}

After evaluating the performance of different endoscopes, we found the optimal endoscope for the highest efficiency of CL collection and then assembled a sensitivity- improved CLE system. We finally measured and evaluated the performance of the newly assembled CLE system, including the spatial resolution, linearity, and sensitivity (minimum detection activity of the radionuclides). In the performance evaluation experiments, the radionuclides of ${ }^{68} \mathrm{Ga}$ were used as the emission source for CL light. This was due to two main considerations. First, in the published work chosen as a reference $(23),{ }^{68} \mathrm{Ga}$ was selected to investigate the system performance, allowing direct comparison between our study and the reference. Second, ${ }^{68} \mathrm{Ga}$ has been used in clinical diagnostics; for example, ${ }^{68} \mathrm{Ga}$-FAPI was reported in the clinical diagnosis of malignancies of the lower gastrointestinal tract $(36,37)$. 
We specially designed a resolution test board suitable for CL resolution measurement of the CLE system. The test board was made of aluminum and painted black to avoid interference from reflections. Groups of evenly distributed lines were drilled into the test board. In each group, the width of the lines was the same as the width of the interval between the edges of the 2 lines. The line widths used in our experiments were $0.6,0.8,1.0$, and $1.2 \mathrm{~mm}$. Approximately $25 \mu \mathrm{Ci}$ of ${ }^{68} \mathrm{Ga}$ was injected into the concave lines of the test board, which was then placed $1 \mathrm{~cm}$ below the endoscope probe for data acquisition. The white-light image was first collected with an exposure time of $0.1 \mathrm{~s}$ and a binning value of 1 . The CL image was then captured with an exposure time of $300 \mathrm{~s}$ and a binning value of 4 .

A single well in a black 96-well plate containing $300 \mu \mathrm{L}$ of saline mixed with $10 \mu \mathrm{Ci}$ of ${ }^{68} \mathrm{Ga}$ was placed $1 \mathrm{~cm}$ below the endoscope probe. The CLE images were sequentially acquired at fixed intervals. During data acquisition, the parameters of the EMCCD camera were set at a binning value of 4 and an EM value of 1,000. The total data acquisition time was around $780 \mathrm{~min}$, approximately 11.5 half-lives of ${ }^{68} \mathrm{Ga}$. To make comparisons with the CLE system used in the previous study (24), we first acquired an image every $15 \mathrm{~min}$, with an image acquisition time of $300 \mathrm{~s}$. To further investigate the performance improvement of the CLE system presented within this study, we also acquired an image every $10 \mathrm{~min}$, with an image acquisition time of 180 , 120,60 , and $30 \mathrm{~s}$. To provide additional guidance for clinical applications, we measured the detection sensitivity of the developed CLE system using the ${ }^{18} \mathrm{~F}-\mathrm{FDG}$ solution as a CL light emission source. The CLE images emitted from ${ }^{18} \mathrm{~F}$ were acquired with an image acquisition time of $300 \mathrm{~s}$.

The 96-well plate-based in vitro experiments adequately evaluated the detection performance of the CLE system under ideal conditions. However, in clinical applications, gastrointestinal lesions are usually located under the mucosal layer, and therefore the CL light needs to pass through this layer in order to be collected by the CLE system. Thus, we conducted further pseudotumor experiments to measure the in vivo detection sensitivity. The ${ }^{68} \mathrm{Ga}$ of $15 \mu \mathrm{Ci}$ was diluted to $50 \mu \mathrm{L}$ with normal saline to form the ${ }^{68} \mathrm{Ga}$ solution. The pseudotumor was constructed with a mixture made of Matrigel (BD Biosciences) and ${ }^{68} \mathrm{Ga}$ solution at a ratio of $1: 1$. The $100 \mu \mathrm{L}$ mixture was injected subcutaneously into the right flank of a hairless mouse. The mouse was then placed on a warm platform for 3 min until the Matrigel solidified. The CLE images were sequentially acquired every $10 \mathrm{~min}$, with an image acquisition time of
300 and $60 \mathrm{~s}$. The other parameters were the same as those of in vitro sensitivity measurements.

The pseudotumor mouse model-based imaging experiments were performed under a project license granted by the Fourth Military Medical University Animal Studies Committee (no. KY20163349-1) in compliance with the Fourth Military Medical University Animal Studies Committee guidelines for the care and use of animals.

\section{Data processing}

Because the radionuclides undergo nuclear decay over time, time-related decay correction was applied for images that were collected continuously over time. In the comparison experiments of different SP distances and different optical fiber endoscopes, the final imaging results were normalized to $150 \mu \mathrm{Ci}$ for investigation of SP distance and $300 \mu \mathrm{Ci}$ for comparison of different endoscopes. In the experiments of sensitivity measurements, the collected images were decay-calibrated based on initial radionuclide activity and acquisition time.

Heavy impulse noise was generated on the EMCCD by high-energy gamma rays of radionuclides during image acquisition. Here, the median filter with a template size of $5 \times 5$ was used to remove these strong noise points. A region of interest (ROI) was outlined automatically by setting a threshold determined empirically through experiments. The mean and standard deviation of the averaged signal intensity in the ROI were used in the following plots. Background subtraction was applied before analysis. An area outside the ROI was selected, which was approximately the same size as the ROI, and the mean intensity value of this area was calculated as the background value. This background value was subtracted from the image for background subtraction. All data processing algorithms were self-written with Matlab 2013b software (MathWorks).

\section{Results}

\section{Influence of the monofilament diameters of the imaging fiber bundle}

Figure $2 A-2 C$ present the CLE images acquired by endoscopes $\mathrm{A}, \mathrm{B}$, and $\mathrm{C}$, the results of which were used to investigate the influence of MF diameters of the imaging fiber bundle on collection efficiency of the CLE system. An obvious difference can be observed among the 3 endoscopes from the comparison maps. The CLE image 

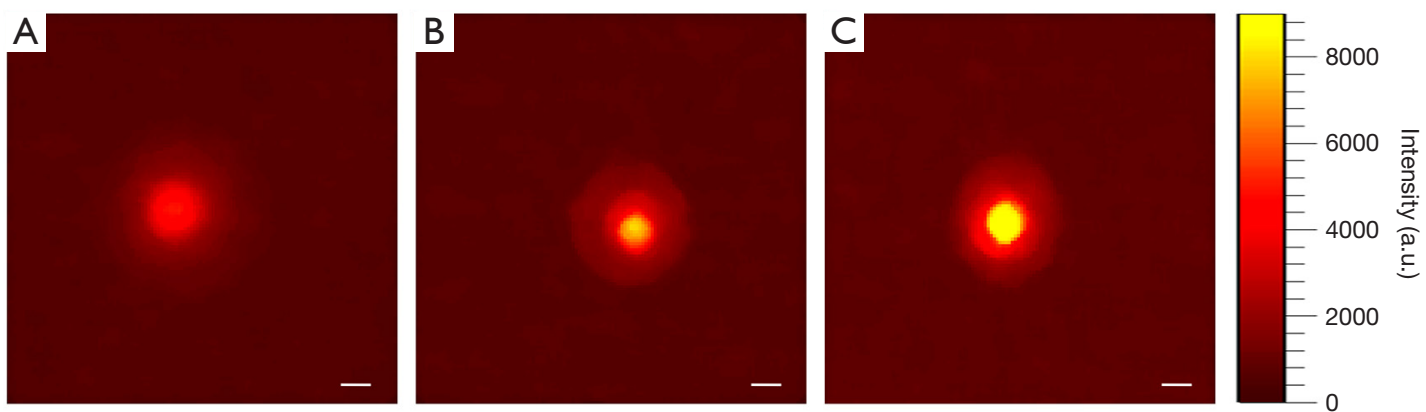

D

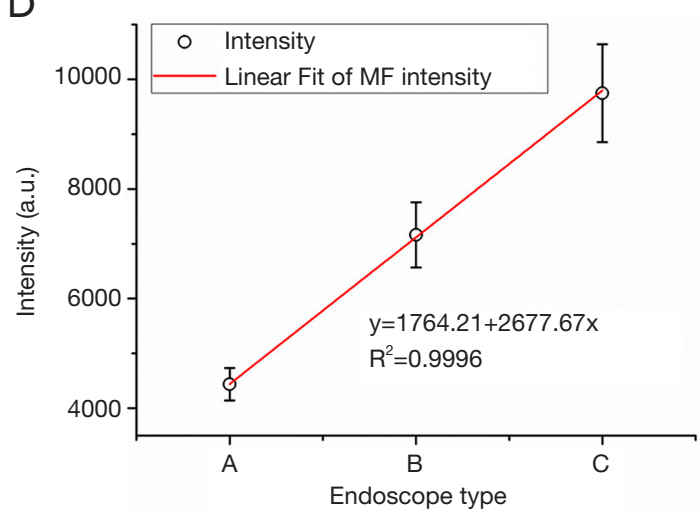

$\mathrm{E}$

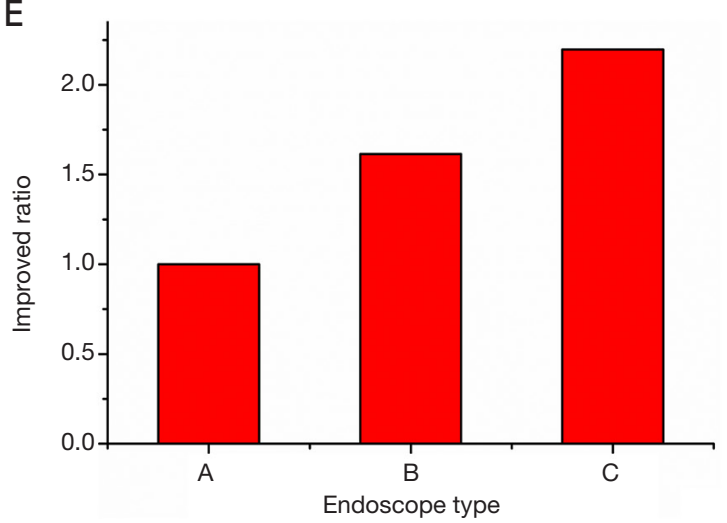

Figure 2 Influence of monofilament (MF) diameters of the imaging fiber bundle on the collection efficiency of the CLE system. (A-C) The CLE images collected by using the A, B, and C endoscopes. (D) Quantitative comparison between 3 endoscopes. (E) Improvement ratio of the $\mathrm{B}$ and $\mathrm{C}$ endoscopes compared to endoscope A. Here, the ${ }^{18} \mathrm{~F}-\mathrm{FDG}$ solution was used as the Cerenkov luminescence light emission source. Scale bar: $4 \mathrm{~mm}$. CLE, Cerenkov luminescence endoscope.

acquired by endoscope $\mathrm{C}$ had the strongest brightness, followed by endoscopes B and A. In order to quantitatively evaluate the difference, we calculated the mean intensity of the ROI in each image and plotted them in Figure 2D). We found that the highest signal intensity was obtained by endoscope $\mathrm{C}$ while the lowest was obtained by endoscope A. Furthermore, a linear increase in the light collection efficiency of the CLE system was obtained as the MF diameter increased $\left(\mathrm{R}^{2}=0.9996\right)$. To intuitively evaluate the improvement on collection efficiency of the CLE system, we set the signal intensity of endoscope $\mathrm{A}$ as the baseline and then calculated the improvement ratio by dividing the signal intensity of the other endoscopes by this value, as plotted in Figure 2E. The CLE system configured with endoscope $\mathrm{C}$ provided the best-improved collection efficiency, with an improvement ratio of 2.20. The results collectively showed that the larger the MF diameter was, the better the collection efficiency of the CLE system.

By calculating the smallest line pair area that can be clearly distinguished in the captured image of the resolution test board, we obtained the white-light spatial resolution of the CLE. We found that the white-light spatial resolution of the CLE system decreases slightly as the MF increases, from a spatial resolution of $0.25 \mathrm{~mm}$ at $20 \mu \mathrm{m}$ to $0.35 \mathrm{~mm}$ at $30 \mu \mathrm{m}$. Such a decrease in white-light spatial resolution is negligible compared to the improvement in the detection efficiency of the system.

\section{Influence of the fiber materials and probe coating}

Figure $3 A, 3 B$ present the CLE images acquired by endoscopes $\mathrm{C}$ and $\mathrm{D}$, the results of which were used to investigate the influence of the fiber material and probe coating on collection efficiency of the CLE system. We found that the image acquired by the CLE system equipped with endoscope $\mathrm{D}$ was much brighter than that acquired with endoscope C. Similarly, we calculated the mean intensity of each image and the corresponding improvement ratio, as plotted in Figure 3C,3D. The CLE system configured with endoscope $\mathrm{D}$ provided higher signal intensity and better signal collection efficiency. The improvement ratio was about 1.84 compared with that equipped with endoscope C. 

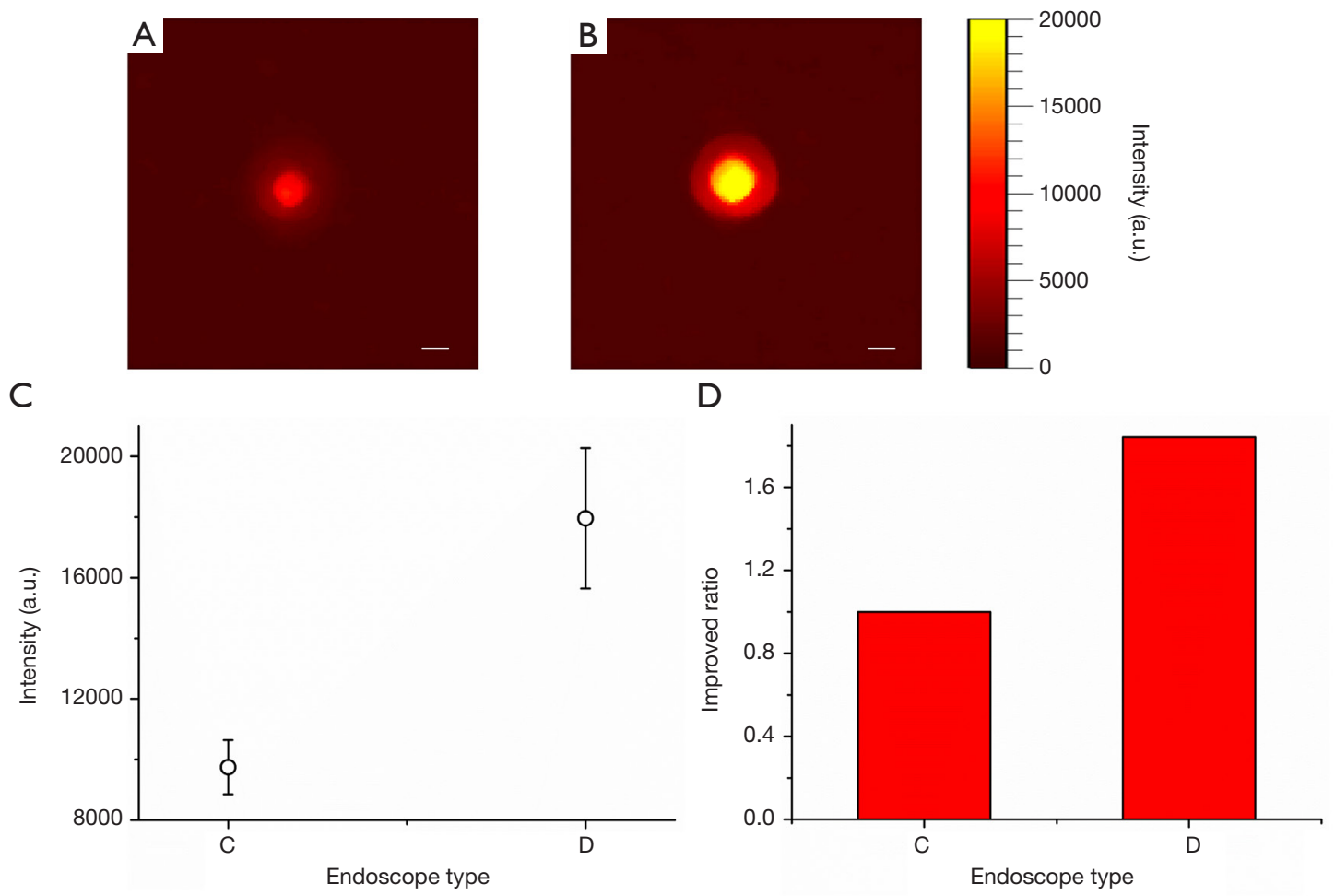

Figure 3 Influence of the fiber materials and probe coating on collection efficiency of the CLE system. The CLE images collected by endoscope C (A) and endoscope D (B). (C) Quantitative comparison between 2 endoscopes. (D) Improvement ratio of endoscope D compared to endoscope C. Here, the ${ }^{18} \mathrm{~F}$-FDG solution was used as the Cerenkov luminescence light emission source. Scale bar: 4 mm. CLE, Cerenkov luminescence endoscope.

These results proved that the endoscope, made from silica glass and having a probe coating suitable for broad spectrum transmission from ultraviolet to near infrared light, can improve the collection efficiency of the CLE system.

\section{Influence of the source-probe distances}

Figure $4 A$ shows the CLE images acquired at different SP distances. We found that with the increase of SP distance, the signal intensity initially changed little before rapidly decreasing after a certain distance. We further calculated the average intensity of the ROI in each image and then plotted the relationship between the average intensity and the SP distance, as drawn in Figure 4B. As SP distance increased, a significant and sharp drop in signal strength was observed when this distance was greater than $1 \mathrm{~cm}$. The average intensity of the detected signal was approximately linearly proportional to the SP distance. When this distance was less than $1 \mathrm{~cm}$, we found that the strength of the detected signal did not decrease with distance, but showed a fluctuation of first increasing and then decreasing. This phenomenon is presumed to be caused by two reasons. First, the CLE cannot focus the radioactive source onto the detector when the SP distance is less than $1 \mathrm{~cm}$. Second, if the distance between the probe and the radionuclides is too small, the FOV of the endoscope will greatly reduce, making it impossible for the endoscope to completely cover the radionuclide boundary. Therefore, the amount of radionuclide covered by the FOV of the endoscope is increased as the distance in this segment increases. Consequently, there will be an increase in signal intensity with an increase in the SP distance. To balance the signal collection efficiency and clinical operability, the SP distance was fixed as $1 \mathrm{~cm}$ in the other experiments.

\section{Performance of the sensitivity-improved CLE}

Following the aforementioned investigation experiments, endoscope D was selected as an optimal configuration in this study for the construction of the sensitivity-improved 

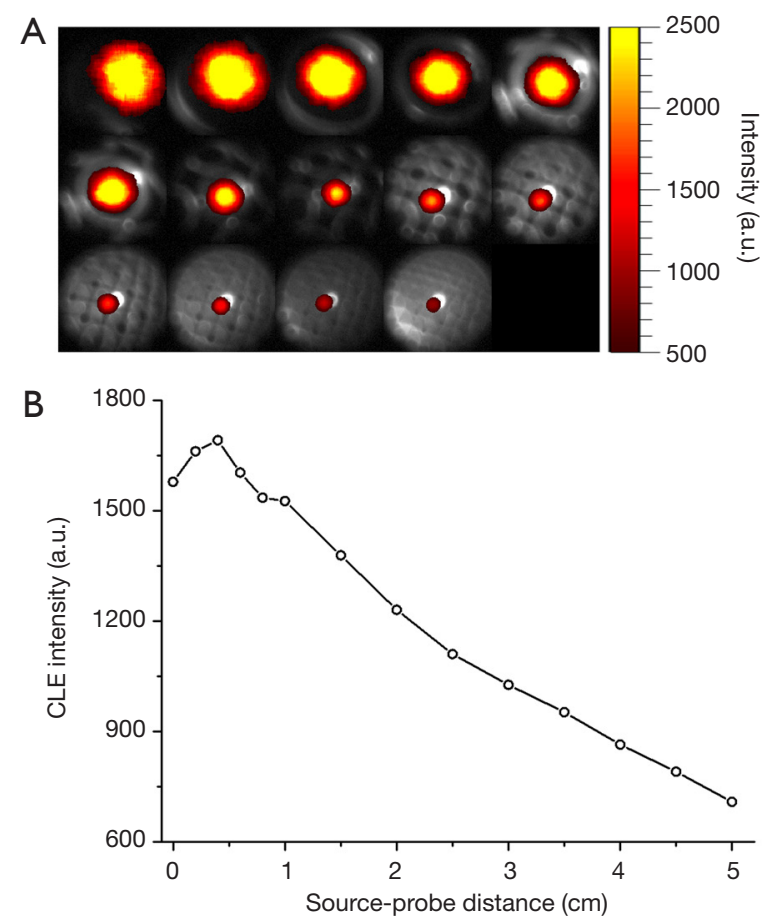

Figure 4 Influence of source-probe distance on signal detection. (A) The CLE images collected at different source-probe distances.

(B) The CLE signal intensity as a function of source-probe distance. Here, the ${ }^{18}$ F-FDG solution was used as the Cerenkov luminescence light emission source. CLE, Cerenkov luminescence endoscope.

CLE system. However, the larger the MF diameter, the more brittle the imaging fiber bundle becomes and the more likely it is to break. Thus, we customized an endoscope that had an imaging fiber bundle with a MF diameter of $28 \mu \mathrm{m}$ and was made of silica glass, and integrated it into the sensitivity-improved CLE system. This MF diameter gave full consideration to the white-light spatial resolution and the maximum light collection efficiency. Additionally, the working distance (SP distance) was fixed at $1 \mathrm{~cm}$.

\section{Fluorescence spatial resolution}

Figure $5 A$ presents the photograph of the specially designed resolution test board, and Figure $5 B$ displays the CLE images fused with the corresponding white-light images for each investigated group of lines. We found that when the line width was not less than $0.8 \mathrm{~mm}$, different lines were clearly distinguished from each other by directly reading the image. There was slight blurring of the CLE image when the line width was $0.6 \mathrm{~mm}$, but low signal strength valleys were still seen between the 3 lines. We extracted
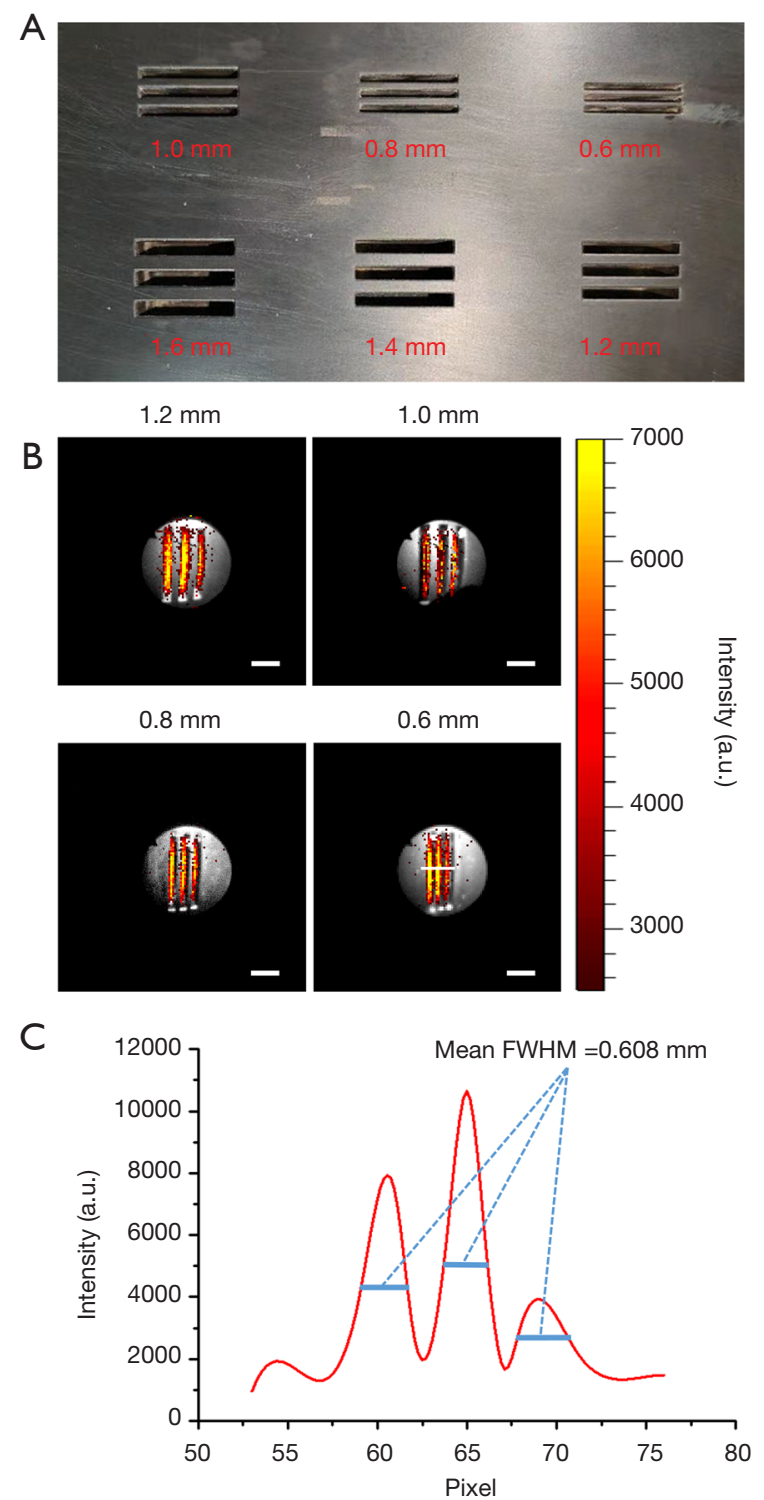

Figure 5 Fluorescence spatial resolution of the highly-sensitive CLE system. (A) Photograph of the specially designed resolution test board. (B) CLE images fused with the corresponding whitelight images for each investigated group of lines. (C) Quantitative line profile (white line in the group of $0.6 \mathrm{~mm}$ line width). Here, the ${ }^{68} \mathrm{Ga}$ was used as the Cerenkov luminescence light emission source. Scale bar in B: 3 mm. CLE, Cerenkov luminescence endoscope.

a line profile along the white line in the CLE image of $0.6 \mathrm{~mm}$ line width, as plotted in Figure $5 \mathrm{C}$. This line profile quantitatively confirmed that the fluorescence spatial resolution of the CLE system was better than $0.6 \mathrm{~mm}$. This spatial resolution was slightly better than that of PET for superficial imaging. Note that the curve in Figure $5 C$ is 
smoother than the heat map in Figure 5B. This is due to the avoidance of high-energy noise points (Figure $5 B$ ) as much as possible when drawing and interpolating this curve.

\section{In vitro sensitivity}

Figure 6 shows the sequential images acquired by using the CLE system over the decay of radionuclides with an image integration time of $300 \mathrm{~s}$. As these images were acquired at different time points, we calibrated these time points to the activity values of the radionuclides based on their decay law. With the decrease of radionuclide activity of ${ }^{68} \mathrm{Ga}$, an apparent trend of intensity attenuation was observed. The minimum resolvable activity we read from the images was $0.27 \mu \mathrm{Ci}$ (circled in yellow). The minimum resolvable activity was defined as the activity corresponding to the point at which the magnitude and distribution of the intensity of the CLE image begins to converge to a constant as the activity decreases. For quantitative analysis, we calculated the average intensity of the CLE signals in the extracted ROIs. The average intensity as a function of radionuclide activity of ${ }^{68} \mathrm{Ga}$ is plotted in Figure $6 \mathrm{~B}$. With linear fitting, a strong linear relationship can be obtained between the average intensity versus the ${ }^{68} \mathrm{Ga}$ activity $\left(\mathrm{R}^{2}=0.9963\right)$. The linear correlation equation indicated that a theoretical minimum activity of ${ }^{68} \mathrm{Ga}$ detected by the CLE system was as low as $0.03 \mu \mathrm{Ci}$. This result was an order of magnitude higher than our previous result (24).

To demonstrate the high sensitivity of the new CLE system, additional time series CLE images were further acquired with a reduced image integration time, including $180,120,60$, and $30 \mathrm{~s}$. The minimum activity at which the images can be resolved was $0.48,1.09,1.53$, and $3.26 \mu \mathrm{Ci}$, respectively. Figure $6 \mathrm{C}-6 \mathrm{~F}$ show the CLE intensity as a function of the radionuclide activity of ${ }^{68} \mathrm{Ga}$ and the corresponding linear fitting results. A strong linear relationship was obtained with an $\mathrm{R}^{2}$ value larger than 0.9500 for all the investigated cases. From the fitting results, we calculated the theoretical minimum detectable activity of ${ }^{68} \mathrm{Ga}$, which were $0.32 \mu \mathrm{Ci}$ at $180 \mathrm{~s}$ image integration time, $0.53 \mu \mathrm{Ci}$ at $120 \mathrm{~s}, 0.68 \mu \mathrm{Ci}$ at $60 \mathrm{~s}$, and $0.82 \mu \mathrm{Ci}$ at $30 \mathrm{~s}$. Furthermore, there was a strong linear relationship between the theoretical minimum detectable activity and the image integration time $\left(\mathrm{R}^{2}=0.9918\right)$, as plotted in Figure 6G. These results collectively revealed that even with the reduced image integration time of $60 \mathrm{~s}$, our new CLE system still has a submicrocurie detection sensitivity.

With similar processing and analysis methods, the minimum resolvable activity of the developed CLE system for ${ }^{18} \mathrm{~F}$ was $1.82 \mu \mathrm{Ci}$ at $300 \mathrm{~s}$ image integration time. The corresponding theoretical minimum detectable activity was $0.72 \mu \mathrm{Ci}$. The detailed results are presented in Figure S2. These results revealed that the developed CLE system also has a submicrocurie detection sensitivity for a radionuclide of ${ }^{18} \mathrm{~F}$.

\section{In vivo sensitivity}

The sequential CLE images over the decay of radionuclides of ${ }^{68} \mathrm{Ga}$ were acquired from the pseudotumor (indicated by red circles and arrows in Figure $7 A$ ) of the mouse model with an image integration time of $300 \mathrm{~s}$, as presented in Figure $7 B$. Similarly, an apparent trend of CLE intensity attenuation was observed with the decrease of the radionuclide activity. The relationship between the average intensity of the extracted ROIs and the activity of ${ }^{68} \mathrm{Ga}$ is depicted in Figure $7 C$. A strong linear relationship was observed $\left(\mathrm{R}^{2}=0.9940\right)$. As seen in Figure $7 B$, we found that the minimum activity at which the image was resolved was $0.93 \mu \mathrm{Ci}$ (circled in yellow). The linear fitting results showed that the theoretical minimum detectable activity was $0.44 \mu \mathrm{Ci}$, which was also an order of magnitude higher than our previous result (24).

To illustrate the high sensitivity of our CLE system and the credibility of its clinical application, we acquired time series CLE images at $60 \mathrm{~s}$ image integration time and explored the minimum radionuclide activity detectable by the system based on these data. Figure $7 D$ shows the time series CLE images, exploring the lowest activity resolvable on the image was smaller than $1.41 \mu \mathrm{Ci}$ (circled in yellow). Similarly, we fitted the relationship between the CLE signal intensity and the radionuclide activity, as shown in Figure $7 E$. The linearity of the fitting result is very strong, with an $\mathrm{R}^{2}$ value of 0.9913. Based on the fitting equation between these results, the theoretical minimum detectable activity was calculated to be as low as $0.83 \mu \mathrm{Ci}$. These results proved that our CLE system achieved a relatively high detection sensitivity at a submicrocurie level in the in vivo measurement.

\section{Discussion}

Cerenkov luminescence endoscope (CLE) integrates a clinical endoscope into the Cerenkov luminescence imaging system, providing promising potential for clinical translation of optical imaging. However, low detection sensitivity limits its clinical applications. In this paper, 4 endoscopes were customized and installed on the CLE system to investigate the influence of the MF fiber diameters of the imaging fiber bundle as well as the fiber material and probe coating on detection efficiency. Results found that larger MF diameter 

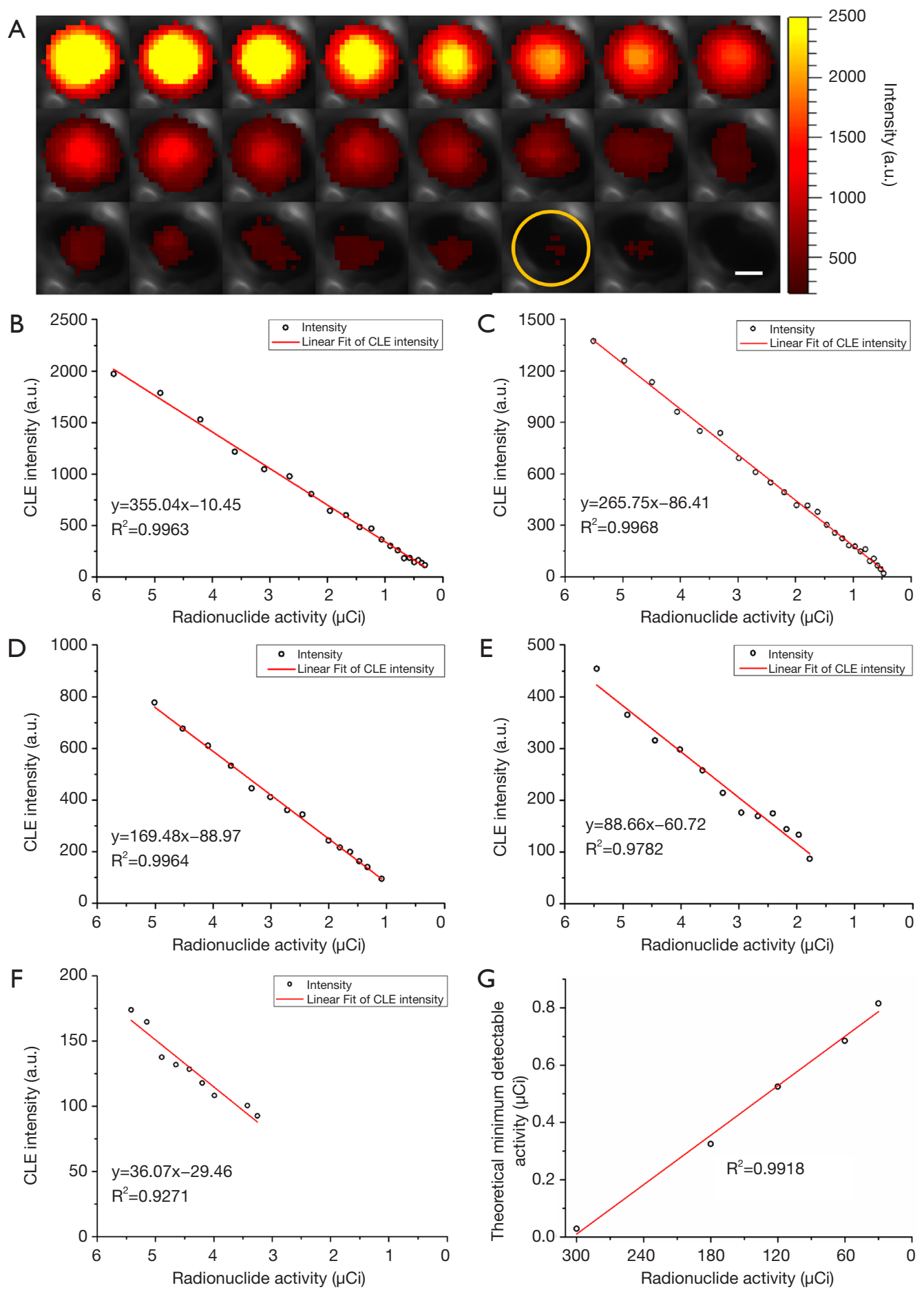

Figure 6 In vitro sensitivity. (A) Sequential images over the decay of radionuclide of ${ }^{68} \mathrm{Ga}$ acquired by the CLE system at image integration time of $300 \mathrm{~s}$. The radionuclide decays from 6.65 to $0.19 \mu \mathrm{Ci}$ from upper left to lower right. (B-F) CLE intensity as a function of the radionuclide activity of ${ }^{68} \mathrm{Ga}$ and corresponding linear fitting results for image integration time of 300, 180, 120, 60, and 30 s, respectively. (G) Linear fitting between the theoretical minimum detectable activity of ${ }^{68} \mathrm{Ga}$ and the image integration time. Scale bar: $2 \mathrm{~mm}$. CLE, Cerenkov luminescence endoscope. 

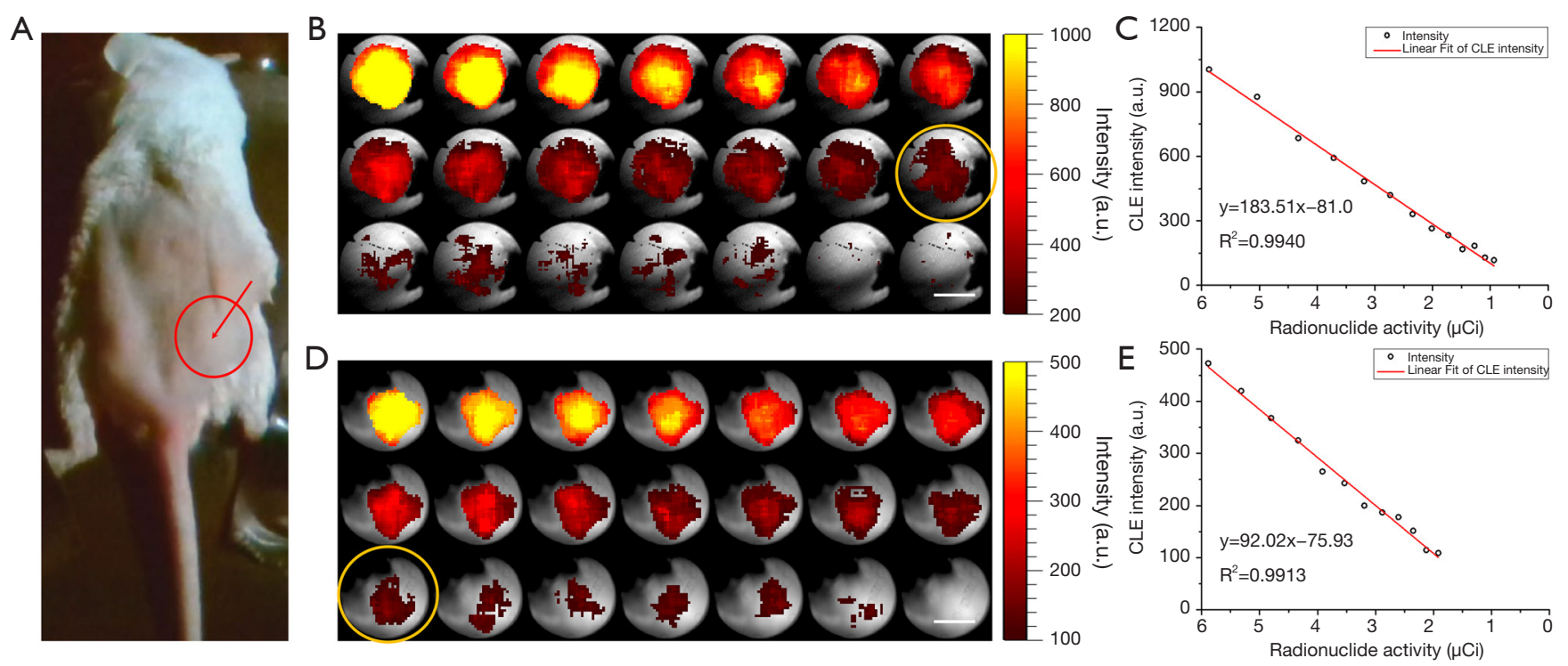

Figure 7 In vivo sensitivity of the sensitivity-improved CLE system. (A) White light image of a pseudotumor mouse model. Sequential images over the decay of radionuclide of ${ }^{68} \mathrm{Ga}$ acquired by the CLE system at the image integration time of $300 \mathrm{~s}$ (B) and $60 \mathrm{~s}$ (C). The radionuclide decays from 6.84 to $0.32 \mu \mathrm{Ci}$ from upper left to lower right in (B), and from 5.89 to $0.77 \mu \mathrm{Ci}$ in (C). (D,E) CLE intensity in (B) and (C) as a function of the radionuclide activity of ${ }^{68} \mathrm{Ga}$ and corresponding linear fitting results. Scale bar: $6 \mathrm{~mm}$. CLE, Cerenkov luminescence endoscope.

and more suitable fiber material and probe coating for CL transmission led to an increased detection efficiency of the CLE system. By using the endoscope with the optimized system parameters, we developed a sensitivity-improved CLE system and measured its sensitivity by imaging ${ }^{68} \mathrm{Ga}$, which showed an enhanced detection sensitivity at a submicrocurie level for both in vitro and in vivo applications. The minimum radioactive activity of radionuclides was as low as $0.03 \mu \mathrm{Ci}$ for in vitro measurements and $0.44 \mu \mathrm{Ci}$ for in vivo measurements, being an order of magnitude higher than the current CLE system (23).

The main obstacle to the clinical application of the current CLE technology is the long image acquisition time required to ensure adequate sensitivity. Therefore, providing high detection sensitivity with a short image acquisition time is the primary challenge that needs to be addressed in early detection of gastric cancer with CLE technology. With an image integration time of $60 \mathrm{~s}$, the in vivo detection sensitivity of ${ }^{68} \mathrm{Ga}$ measured from the pseudotumor mouse model was around $0.83-1.41 \mu \mathrm{Ci}$. Generally, gastric cancer originates from the mucosa of the gastrointestinal tract. Thus, CLE imaging of subcutaneous pseudotumor in hairless mice can adequately mimic the endoscopic detection of gastric cancer. According to the American Joint Committee on Cancer
(AJCC) cancer staging manual, tumors less than $2 \mathrm{~cm}$ in diameter are defined as early tumors (38). To distinguish between the benign and malignant tumors in clinical settings, the standardized uptake value of radionuclides in the positive tumor area should not be less than 3 . Considering the radionuclides dosage commonly injected into clinical patients, the concentration of radionuclides that accumulate in the positive tumor area should be greater than $0.3 \mu \mathrm{Ci} / \mathrm{mL}$. Thus, with the above in vivo detection sensitivity, it is clinically possible to detect a gastric tumor of 1.4 to $1.67 \mathrm{~cm}$ in diameter within $1 \mathrm{mi}$ using the proposed CLE system. This is a considerable improvement to the clinical application of CLE technology.

There are issues that need to be addressed before the CLE system can be introduced to clinical environments. First, both the benefits and drawbacks of the use of a radioactive source-based probe need to be considered. ${ }^{18} \mathrm{~F}$-FDG as a type of radioactive source that can reflect metabolic capacity has been widely used for detection of early-stage cancer in clinics. However, the proposed CLE system is not highly sensitive in detecting ${ }^{18} \mathrm{~F}$, making it difficult to apply this method to the diagnosis of early gastric cancer. As mentioned above, the detection sensitivity of the proposed CLE system for ${ }^{68} \mathrm{Ga}$ can reach 
a submicrocurie level with an image acquisition time of 1 minute, which realizes the detection of early gastric tumor with a diameter of about $1.5 \mathrm{~cm} .{ }^{68} \mathrm{Ga}$ has also been used in clinic, including in the diagnosis of gastric cancer. For example, the ${ }^{68} \mathrm{Ga}$ labeled fibroblast activation protein inhibitor $\left({ }^{68} \mathrm{Ga}-\mathrm{FAPI}\right)$ has been used in the clinical diagnosis of malignancies of the lower gastrointestinal tract $(36,37)$.

A second issue involves the requirement of the imaging device to be approved by a designated agency. The CLE system presented within this study is a new imaging device with components that need to be inserted inside the human body. Thus, the system will need to undergo a series of additional experiments, such as a biosafety assessment and preclinical validation. A license for clinical use is also needed before the device can be used in clinic. Although this may be a lengthy process, we believe that the use of this technology within a clinical setting can significantly improve the outcome of clinical gastrointestinal diseases.

In the evaluation experiments of different endoscopes, when the MF diameter increased from 20 to $30 \mu \mathrm{m}$, the average signal intensity increased linearly by 2.20 . This indicates that detection efficiency can be further improved by a greater increase in MF diameter. However, the spatial resolution decrease as the monofilament diameter increases. Furthermore, if the MF diameter is too large, the flexibility of the optical fiber bundle will be poor and therefore unsuitable for clinical use. Thus, a compromise was decided on for the level of performance with the endoscope set at a MF diameter of $28 \mu \mathrm{m}$ for the CLE system. When changing the fiber materials to silica glass, the detection efficiency of the CLE system was also increased, resulting in an almost 2 -fold improvement. Theoretically, improvement of the fiber material and probe coating properties to increase the transmittance values would further enhance the detection performance of the CLE system.

We also investigated the working temperature of the EMCCD camera to determine the effects of dark current noise on image quality. The working temperature was controlled by changing the temperature from 0 to $-85^{\circ} \mathrm{C}$ (Figure S3). Results showed that the trend of dark current noise decreased when the temperature dropped below $-50^{\circ} \mathrm{C}$. In our experiments, the EMCCD camera was cooled to $-85^{\circ} \mathrm{C}$ to allow it to function at a dark-current-noiselimited condition. Although the working temperature could be decreased to $-100{ }^{\circ} \mathrm{C}$, this had little effect on improving image quality. In addition, we explored the effects of scintillation effects arising from the interaction of high-energy radioactive particles with optical fibers. By comparing CLE images acquired with and without the radionuclide source covered by black cardboard (detailed in Figure S4), we found that the influence of the scintillation effect on image acquisition was negligible under the experimental conditions of this study.

There is still room for further improvement of the CLE system, such as the use of a more suitable detector. As mentioned earlier, Cerenkov luminescence light has unique spectral properties. The wide spectrum ranges from ultraviolet to near-infrared wavelength, with most of the energy located at the region of lower wavelengths. The ongoing study aimed to improve the detection efficiency of the CLE system by identifying a detector sensor with higher sensitivity at the lower wavelength region. It should also be noted that the FOV of the optical fiber endoscope occupies a small circular space on the EMCCD, an area roughly onesixteenth of the size of the pixels occupied by the effective detection area of the EMCCD. This results in a very small effective area in which the EMCCD can be used. In order to present the results clearly, the images in Figures 4,6, and 7 were cropped to retain only the endoscopic image region. Through our series of experiments, we found that it is possible to increase the number of pixels occupied by the endoscopic image on the EMCCD by extending the length of the SCD, but this in turn causes a reduction in the efficiency of signal detection. Increasing the use of the EMCCD pixel area while maintaining the efficiency of signal detection is an issue which is currently being addressed.

\section{Conclusions}

In summary, with the goal of improving the detection sensitivity of the current CLE system to allow for greater clinical applications we developed a highly sensitive CLE system by optimizing structural parameters. The series of experimental results showed that the detection sensitivity of the system was able to reach a submicrocurie level at $1 \mathrm{~min}$ image acquisition time. This verifies the ability of this technology to detect early tumors within $1 \mathrm{~min}$ in clinical settings and brings to light a promising potential method for the diagnosis of clinical gastrointestinal diseases.

\section{Acknowledgments}

Xueli Chen would like to thank Dr. Fei Kang and Prof. Jing Wang for their help in preparing radionuclide sources and supporting the experiments.

Funding: This work was supported in part by the National 
Natural Science Foundation of China (no. 81627807, 81871397, and 91959208), the National Young Top-notch Talent of "Ten Thousand Talents Program", the Shaanxi Science Fund for Distinguished Young Scholars (no. 2020JC-27), the Fok Ying-Tung Education Foundation of China (161104), and the Shaanxi Young Top-notch Talent of "Special Support Program".

\section{Footnote}

Conflicts of Interest: All authors have completed the ICMJE uniform disclosure form (available at https://dx.doi. org/10.21037/qims-21-373). The authors have no conflicts of interest to declare.

Ethical Statement: The authors are accountable for all aspects of the work in ensuring that questions related to the accuracy or integrity of any part of the work are appropriately investigated and resolved. Experiments were performed under a project license granted by the Fourth Military Medical University Animal Studies Committee (no. KY20163349-1), in compliance with the Fourth Military Medical University Animal Studies Committee guidelines for the care and use of animals.

Open Access Statement: This is an Open Access article distributed in accordance with the Creative Commons Attribution-NonCommercial-NoDerivs 4.0 International License (CC BY-NC-ND 4.0), which permits the noncommercial replication and distribution of the article with the strict proviso that no changes or edits are made and the original work is properly cited (including links to both the formal publication through the relevant DOI and the license). See: https://creativecommons.org/licenses/by-nc-nd/4.0/.

\section{References}

1. Bray F, Ferlay J, Soerjomataram I, Siegel RL, Torre LA, Jemal A. Global cancer statistics 2018: GLOBOCAN estimates of incidence and mortality worldwide for 36 cancers in 185 countries. CA Cancer J Clin 2018;68:394-424.

2. Chen W, Zheng R, Baade PD, Zhang S, Zeng H, Bray F, Jemal A, Yu XQ, He J. Cancer statistics in China, 2015. CA Cancer J Clin 2016;66:115-32.

3. Mazzei MA, Di Giacomo L, Bagnacci G, Nardone V, Gentili F, Lucii G, Tini P, Marrelli D, Morgagni P, Mura G, Baiocchi GL, Pittiani F, Volterrani L, Roviello F. Deltaradiomics and response to neoadjuvant treatment in locally advanced gastric cancer-a multicenter study of GIRCG (Italian Research Group for Gastric Cancer). Quant Imaging Med Surg 2021;11:2376-87.

4. Weissleder R, Mahmood U. Molecular imaging. Radiology 2001;219:316-33.

5. Pysz MA, Gambhir SS, Willmann JK. Molecular imaging: current status and emerging strategies. Clin Radiol 2010;65:500-16.

6. Wang K, Chi C, Hu Z, Liu M, Hui H, Shang W, Peng D, Zhang S, Ye J, Liu H, Tian J. Optical Molecular Imaging Frontiers in Oncology: The Pursuit of Accuracy and Sensitivity. Engineering 2015;1:309-23.

7. Tang XJ, Zhang WJ, Han YN, et al. Optical imaging technique applied in biological tissue and feasible technique. Chinese Journal of Medical Physics 2000;17:17-20.

8. Ecclestone BR, Abbasi S, Bell K, Dinakaran D, Bigras G, Mackey JR, Haji Reza P. Towards virtual biopsies of gastrointestinal tissues using photoacoustic remote sensing microscopy. Quant Imaging Med Surg 2021;11:1070-7.

9. Kuchimaru T, Iwano S, Kiyama M, Mitsumata S, Kadonosono T, Niwa H, Maki S, Kizaka-Kondoh S. A luciferin analogue generating near-infrared bioluminescence achieves highly sensitive deep-tissue imaging. Nat Commun 2016;7:11856.

10. Kircher MF, de la Zerda A, Jokerst JV, Zavaleta CL, Kempen PJ, Mittra E, Pitter K, Huang R, Campos C, Habte F, Sinclair R, Brennan CW, Mellinghoff IK, Holland EC, Gambhir SS. A brain tumor molecular imaging strategy using a new triple-modality MRI-photoacoustic-Raman nanoparticle. Nat Med 2012;18:829-34.

11. Cerenkov PA. Visible luminescence of pure liquids under action of $\gamma$-radiation. Dok Aka Nau 1934;(2):451-4.

12. Robertson R, Germanos MS, Li C, Mitchell GS, Cherry SR, Silva MD. Optical imaging of Cerenkov light generation from positron-emitting radiotracers. Phys Med Biol 2009;54:N355-65.

13. Ruggiero A, Holland JP, Lewis JS, Grimm J. Cerenkov luminescence imaging of medical isotopes. J Nucl Med 2010;51:1123-30.

14. Robertson R, Germanos MS, Manfredi MG, Smith PG, Silva MD. Multimodal imaging with (18)F-FDG PET and Cerenkov luminescence imaging after MLN4924 treatment in a human lymphoma xenograft model. J Nucl Med 2011;52:1764-9.

15. Tanha K, Pashazadeh AM, Pogue BW. Review of biomedical Čerenkov luminescence imaging applications. Biomed Opt Express 2015;6:3053-65.

16. Cai M, Zhang Z, Shi X, Yang J, Hu Z, Tian J. Non-Negative 
Iterative Convex Refinement Approach for Accurate and Robust Reconstruction in Cerenkov Luminescence Tomography. IEEE Trans Med Imaging 2020;39:3207-17.

17. Spinelli AE, Ferdeghini M, Cavedon C, Zivelonghi E, Calandrino R, Fenzi A, Sbarbati A, Boschi F. First human Cerenkography. J Biomed Opt 2013;18:20502.

18. Thorek DL, Riedl CC, Grimm J. Clinical Cerenkov luminescence imaging of (18)F-FDG.J Nucl Med 2014;55:95-8.

19. Grootendorst MR, Cariati M, Pinder SE, Kothari A, Douek M, Kovacs T, et al. Intraoperative Assessment of Tumor Resection Margins in Breast-Conserving Surgery Using 18F-FDG Cerenkov Luminescence Imaging: A First-inHuman Feasibility Study. J Nucl Med 2017;58:891-8.

20. Darr C, Krafft U, Fendler WP, Costa PF, Barbato F, Praus C, Reis H, Hager T, Tschirdewahn S, Radtke JP, Herrmann K, Hadaschik BA. First-in-man intraoperative Cerenkov luminescence imaging for oligometastatic prostate cancer using 68Ga-PSMA-11. Eur J Nucl Med Mol Imaging 2020;47:3194-5.

21. Kothapalli SR, Liu H, Liao JC, Cheng Z, Gambhir SS. Endoscopic imaging of Cerenkov luminescence. Biomed Opt Express 2012;3:1215-25.

22. Liu H, Carpenter CM, Jiang H, Pratx G, Sun C, Buchin MP, Gambhir SS, Xing L, Cheng Z. Intraoperative imaging of tumors using Cerenkov luminescence endoscopy: a feasibility experimental study. J Nucl Med 2012;53:1579-84.

23. Cao X, Chen X, Kang F, Lin Y, Liu M, Hu H, Nie Y, Wu K, Wang J, Liang J, Tian J. Performance evaluation of endoscopic Cerenkov luminescence imaging system: in vitro and pseudotumor studies. Biomed Opt Express 2014;5:3660-70.

24. Hu H, Cao X, Kang F, Wang M, Lin Y, Liu M, Li S, Yao L, Liang J, Liang J, Nie Y, Chen X, Wang J, Wu K. Feasibility study of novel endoscopic Cerenkov luminescence imaging system in detecting and quantifying gastrointestinal disease: first human results. Eur Radiol 2015;25:1814-22.

25. Song T, Liu X, Qu Y, Liu H, Bao C, Leng C, Hu Z, Wang K, Tian J. A Novel Endoscopic Cerenkov Luminescence Imaging System for Intraoperative Surgical Navigation. Mol Imaging 2015;14:443-9.

26. Carpenter CM, Ma X, Liu H, Sun C, Pratx G, Wang J, Gambhir SS, Xing L, Cheng Z. Cerenkov luminescence endoscopy: improved molecular sensitivity with $\beta$-emitting radiotracers. J Nucl Med 2014;55:1905-9.

27. Sun C, Pratx G, Carpenter CM, Liu H, Cheng Z, Gambhir SS, Xing L. Synthesis and radioluminescence of PEGylated Eu(3+) -doped nanophosphors as bioimaging probes. Adv Mater 2011;23:H195-9.

28. Thorek DL, Ogirala A, Beattie BJ, Grimm J. Quantitative imaging of disease signatures through radioactive decay signal conversion. Nat Med 2013;19:1345-50.

29. King M, Carpenter C, Sun C, Pratx G, Xing L. Feasibility evaluation of radioluminescence for oncologic imaging. $J$ Nucl Med 2014;55.

30. Cao X, Chen X, Kang F, Zhan Y, Cao X, Wang J, Liang J, Tian J. Intensity Enhanced Cerenkov Luminescence Imaging Using Terbium-Doped Gd2O2S Microparticles. ACS Appl Mater Interfaces 2015;7:11775-82.

31. Cao X, Chen X, Kang F, Cao X, Zhan Y, Wang J, Wu K, Liang J. Sensitivity improvement of Cerenkov luminescence endoscope with terbium doped Gd2O2S nanoparticles. Appl Phys Lett 2015;106:213702.

32. Hu Z, Qu Y, Wang K, Zhang X, Zha J, Song T, Bao C, Liu H, Wang Z, Wang J, Liu Z, Liu H, Tian J. In vivo nanoparticle-mediated radiopharmaceutical-excited fluorescence molecular imaging. Nat Commun 2015;6:7560.

33. Shaffer TM, Pratt EC, Grimm J. Utilizing the power of Cerenkov light with nanotechnology. Nat Nanotechnol 2017;12:106-17.

34. Ferreira CA, Ni D, Rosenkrans ZT, Cai W. RadionuclideActivated Nanomaterials and Their Biomedical Applications. Angew Chem Int Ed Engl 2019;58:13232-52.

35. Qiu J, Liu Y, Xia Y. Radiolabeling of Gold Nanocages for Potential Applications in Tracking, Diagnosis, and ImageGuided Therapy. Adv Healthc Mater 2021. [Epub ahead of print]. doi: 10.1002/adhm.202002031.

36. Koerber SA, Staudinger F, Kratochwil C, Adeberg S, Haefner MF, Ungerechts G, Rathke H, Winter E, Lindner T, Syed M, Bhatti IA, Herfarth K, Choyke PL, Jaeger D, Haberkorn U, Debus J, Giesel FL. The Role of 68Ga-FAPI PET/CT for Patients with Malignancies of the Lower Gastrointestinal Tract: First Clinical Experience. J Nucl Med 2020;61:1331-6.

37. Chen H, Zhao L, Ruan D, Pang Y, Hao B, Dai Y, Wu X, Guo W, Fan C, Wu J, Huang W, Lin Q, Sun L, Wu H. Usefulness of $68 \mathrm{GaGa-DOTA-FAPI-04} \mathrm{PET/CT} \mathrm{in}$ patients presenting with inconclusive 18FFDG PET/CT findings. Eur J Nucl Med Mol Imaging 2021;48:73-86.

38. Edge SB, Byrd DR, Compton CC, Fritz AG, Greene FL, Trotti A, III. AJCC cancer staging manual. New York, NY, USA: Springer-Verlag, 2010; 103-115.

Cite this article as: Chen $\mathrm{X}$, Wang $\mathrm{X}$, Yan T, Zheng $\mathrm{Y}$, Cao H, Ren F, Cao X, Meng X, Lu X, Liang S, Wu K. Sensitivity improved Cerenkov luminescence endoscopy using optimal system parameters. Quant Imaging Med Surg 2022;12(1):425-438. doi: 10.21037/qims-21-373 


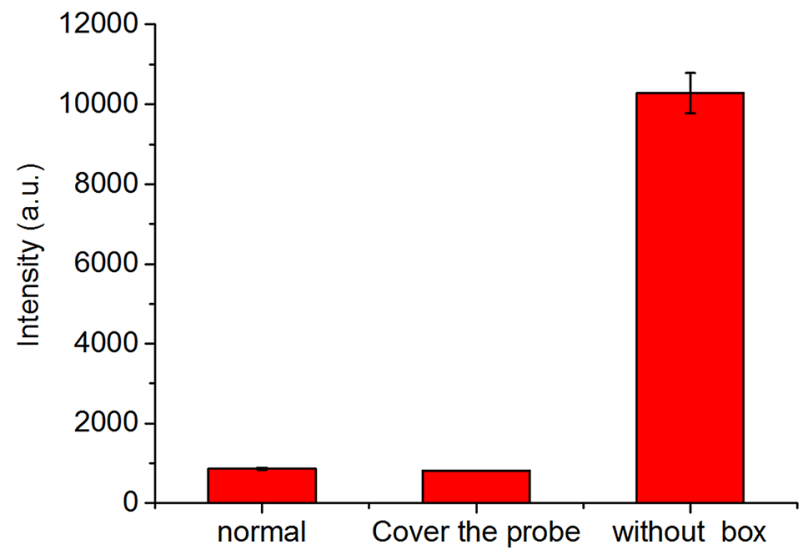

Figure S1 Relevant results of the noise analysis. Three groups of investigations were conducted. The experimental conditions were exactly the same throughout all experiments in this study, labeled as the "normal group" in the figure. The endoscope probe was covered so that no light was able to enter through the probe, which is labeled as the "cover the probe" group in the figure. In the first 2 groups, it was necessary for the light in the data acquisition room to be left off and the endoscope probe to be kept inside the light-tight box during the experimental data acquisition. In the third group, the light-tight box was not used, and the entire CLE system was exposed in the data acquisition room. The light in the data acquisition room was also left off during the experimental data acquisition. The noise level of the first 2 groups was similar and far less than that of the third group. We concluded that the light-tight box played a significant role in isolating ambient light noise. Within real-world clinical application, the human body is substituted for the role of the light-tight box. Thus, such an experimental configuration is similar to the reality of clinical applications. The relevant results showed that the effect of ambient noise in this kind of experimental setup is negligible. CLE, Cerenkov luminescence endoscope. (a)

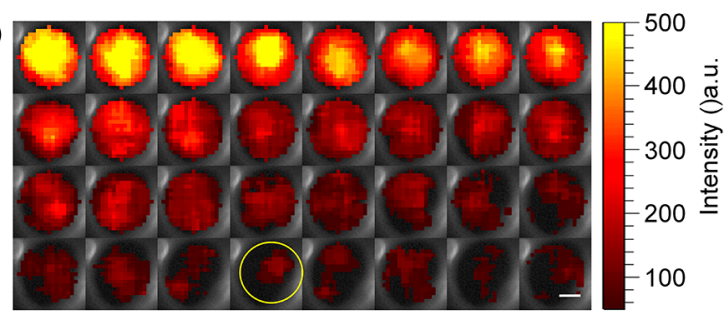

(b)

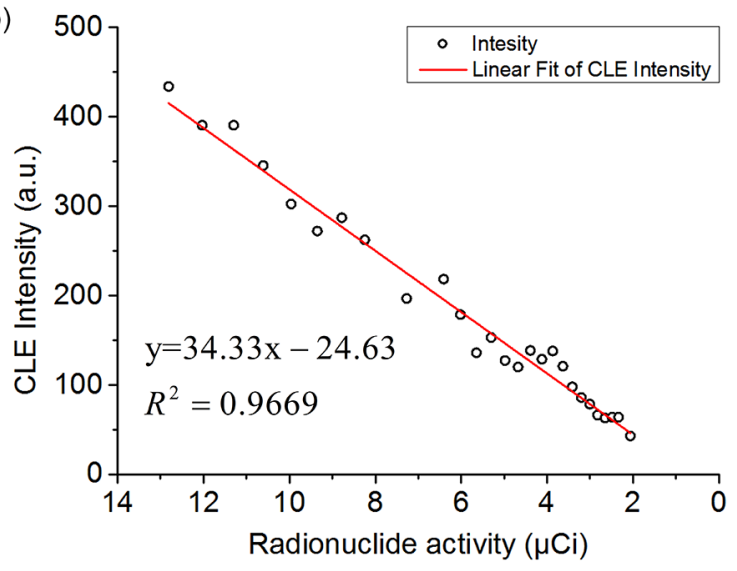

Figure S2 Detection capability of the proposed CLE system for ${ }^{18}$ F-FDG. (A) Sequential images over the decay of radionuclide of ${ }^{18} \mathrm{~F}$ acquired by the CLE system at the image integration time of $300 \mathrm{~s}$. The radionuclide decays from 12.81 to $1.41 \mu \mathrm{Ci}$ from the upper left to lower right. Scale bar: $2 \mathrm{~mm}$. (B) CLE intensity as a function of the radionuclide activity of ${ }^{18} \mathrm{~F}$ and corresponding linear fitting results. (A) The minimum resolvable activity of the developed CLE system for ${ }^{18} \mathrm{~F}$ is $1.82 \mu \mathrm{Ci}$. The corresponding theoretical minimum detectable activity is $0.72 \mu \mathrm{Ci}$. These results revealed that the developed CLE system also had a submicrocurie detection sensitivity for radionuclide of ${ }^{18} \mathrm{~F}$. CLE, Cerenkov luminescence endoscope. 


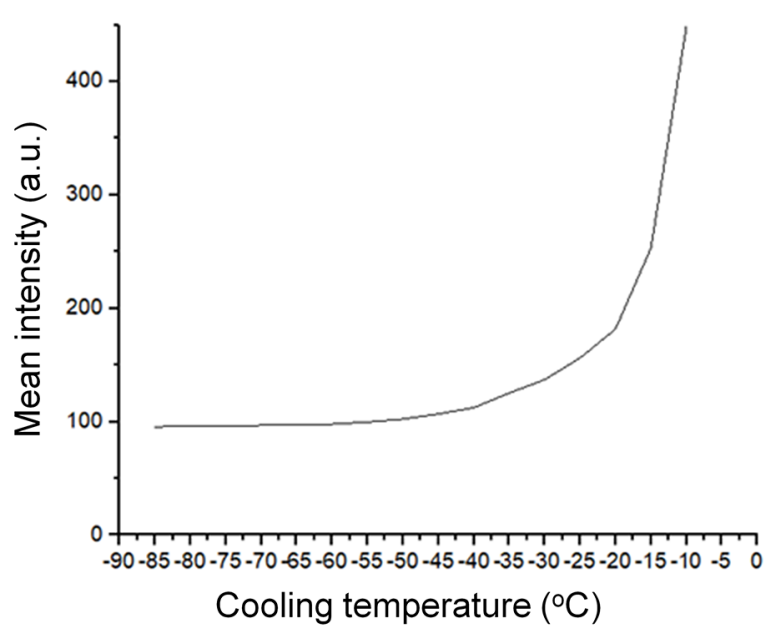

(a)

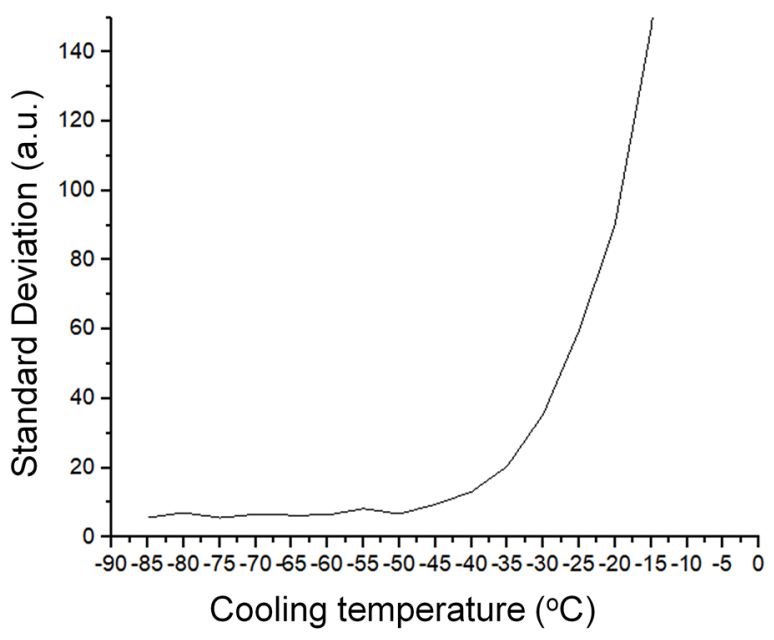

(b)

Figure S3 Effect of the cooling temperature of the EMCCD on the signal collection efficiency. (A,B) show the change of the mean value and standard deviation of EMCCD signal intensity with its cooling temperature. We found that when the cooling temperature of EMCCD was lower than $-50{ }^{\circ} \mathrm{C}$, the mean value and standard deviation of the detected signal tended to be flat. EMCCD, electron multiplying chargecoupled device.

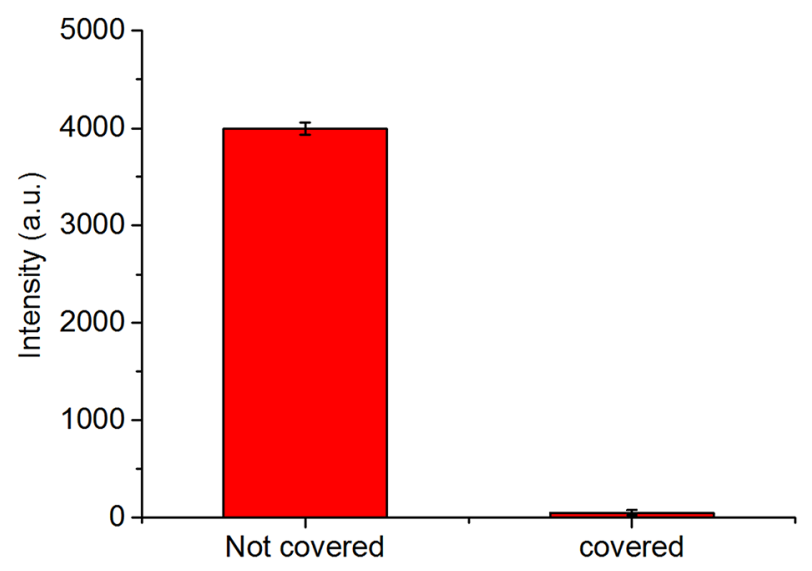

Figure S4 Effects of scintillation effects arising from the interaction of high-energy radioactive particles with optical fibers. In the experiment, we used a piece of black cardboard to cover the radioactive source, which blocked the emission of CL light while still allowing high-energy gamma rays to pass through. We acquired 2 images using the proposed CLE system. One was acquired without the black cardboard covering the radioactive source, labeled as "Not covered" in the figure. The second image was collected with the radioactive source covered with black cardboard, labeled as "covered" in the figure. We found that the intensity of the image signal collected by the CLE system was much lower than that of the image signal without the black cardboard covering. This indicated that the scintillation effect produced by the interaction between high-energy gamma rays and optical fiber was very small. We can thus conclude that the influence of the scintillation effect on image acquisition was negligible under the experimental conditions of this study. CLE, Cerenkov luminescence endoscope. 